\title{
Electromagnetic corrections for the analysis of low energy $\pi^{-} p$ scattering data
}

\author{
A. Gashi ${ }^{a}$ E. Matsinos*a G.C. Oades ${ }^{\text {b }}$ G. Rasche ${ }^{\text {a }}$ \\ W.S. Woolcock ${ }^{\mathrm{c}}$ \\ anstitut für Theoretische Physik der Universität, Winterthurerstrasse 190, \\ CH-8057 Zürich, Switzerland \\ ${ }^{\mathrm{b}}$ Institute of Physics and Astronomy, Aarhus University, DK-8000 Aarhus C, \\ Denmark \\ ${ }^{\mathrm{c}}$ Department of Theoretical Physics, IAS, The Australian National University, \\ Canberra, ACT 0200, Australia \\ * Present address: The KEY Institute for Brain-Mind Research, University \\ Hospital of Psychiatry, Lenggstrasse 31, CH-8029 Zürich, Switzerland.
}

\begin{abstract}
We calculate the electromagnetic corrections to the isospin invariant mixing angle and to the two eigenphases for the $s^{-}, p_{1 / 2^{-}}$and $p_{3 / 2^{-}}$-partial waves for $\pi^{-} p$ elastic and charge exchange scattering. These corrections have to be applied to the nuclear quantities in order to obtain the two hadronic phase shifts for each partial wave. The calculation uses relativised Schrödinger equations containing the sum of an electromagnetic potential and an effective hadronic potential. The mass differences between $\pi^{-}$and $\pi^{0}$ and between $p$ and $n$ are taken into account. We compare our results with those of previous calculations and estimate the uncertainties in the corrections.
\end{abstract}

PACS: 13.75.Gx,25.80.Dj

Key words: $\pi N$ elastic scattering, $\pi N$ electromagnetic corrections, $\pi N$ phase shifts

\section{Introduction}

In the previous paper [1] we described the calculation of the electromagnetic corrections to the three hadronic phase shifts with $l=0,1$ for $\pi^{+} p$ elastic scattering at pion laboratory kinetic energy $T_{\pi} \leq 100 \mathrm{MeV}$, using relativised Schrödinger equations (RSEs) containing the sum of an electromagnetic potential and an effective hadronic potential. In Section 1 of Ref.[1] we gave our 
reasons for using such a potential model rather than the dispersion theory model of the NORDITA group [2,3]. Our aim in this paper is to describe the corresponding calculations for the two-channel $\left(\pi^{-} p, \pi^{0} n\right)$ system in the same energy region.

The phase-shift analysis (PSA) of the low energy $\pi^{+} p$ elastic scattering data and the simultaneous calculation of the electromagnetic corrections was possible because, ignoring the minute inelasticities due to bremsstrahlung, only one real phase shift is needed for each partial wave. Such a programme is not possible for the low energy $\pi^{-} p$ scattering data because three parameters (two eigenphases and a mixing angle), and therefore three electromagnetic corrections, need to be obtained for each partial wave. However, while the set of $\pi^{-} p$ elastic scattering data is only slightly smaller (roughly 300 points) than the set of $\pi^{+} p$ data, there are only 53 published data points for charge exchange scattering in our energy range. It is quite out of the question to extract nine parameters from a PSA of the available data without making further assumptions. The data can reliably yield only one parameter for each partial wave, as in the $\pi^{+} p$ case.

That means that we are forced in the two-channel case to invoke the assumption of isospin invariance for the hadronic interaction in some form. The situation is further complicated by the presence of differences between the physical masses of the charged and neutral particles. Since we keep to the point of view adopted in Ref.[1], that we take account only of those electromagnetic effects that can be calculated with reasonable confidence, we analyse the $\pi^{-} p$ data and calculate the electromagnetic corrections on the assumption that the two-channel system can be treated at the effective hadronic level (which is certainly not the true hadronic situation when the electromagnetic interaction is switched off) as an isospin invariant system with all the pions having the physical mass $\mu_{c}$ of $\pi^{ \pm}$and both $p$ and $n$ having the physical mass $m_{p}$. This implies that the hadronic phase shifts $\delta_{l \pm}^{h}$, obtained from the PSA of the $\pi^{+} p$ data which went hand in hand with the calculation of the electromagnetic corrections, are identified with phase shifts $\left(\delta_{3}^{h}\right)_{l \pm}$ corresponding to total isospin $T=3 / 2$. They can therefore be used as known input to the PSA of the $\pi^{-} p$ data. This PSA will then yield three new hadronic phase shifts $\left(\delta_{1}^{h}\right)_{l \pm}$ corresponding to $T=1 / 2$.

We therefore assume that there are real symmetric $2 \times 2$ matrices $\mathbf{t}_{l \pm}^{h}$ which generalise $\tan \delta_{l \pm}^{h}$ from the $\pi^{+} p$ case to the two channel situation:

$$
\mathbf{t}_{l \pm}^{h}=\mathbf{O}\left(\phi^{h}\right)\left(\begin{array}{cc}
\tan \left(\delta_{1}^{h}\right)_{l \pm} & 0 \\
0 & \tan \left(\delta_{3}^{h}\right)_{l \pm}
\end{array}\right) \mathbf{O}\left(\phi^{h}\right)^{t} .
$$

The orthogonal transformation (1) takes the hadronic $t$-matrices from the 
isospin basis (in which they are diagonal) to the physical basis. The isospin invariant mixing angle is $\phi^{h}=\arcsin (1 / \sqrt{3})$ and

$$
\mathbf{O}(\phi)=\left(\begin{array}{cc}
\cos \phi & \sin \phi \\
-\sin \phi & \cos \phi
\end{array}\right)
$$

In order to analyse the low energy $\pi^{-} p$ scattering data it is necessary to calculate electromagnetic correction matrices $\mathbf{t}_{l \pm}^{e m}$ which, when added to the $\mathbf{t}_{l \pm}^{h}$, give the real symmetric $2 \times 2$ nuclear matrices $\mathbf{t}_{l \pm}^{n}$ :

$$
\mathbf{t}_{l \pm}^{n}=\mathbf{t}_{l \pm}^{h}+\mathbf{t}_{l \pm}^{e m}
$$

The experimental observables are related to the nuclear partial wave matrices $\mathbf{t}_{l \pm}^{n}$ by formulae which are given in Section 2 and in Eqs.(1,2) of Ref.[2].

For the calculation of the $\mathbf{t}_{l \pm}^{e m}$ we use the potential model of Ref.[1] in a generalisation to the two-channel case. We emphasise that the potentials are introduced only in order to calculate the corrections. In addition to the potentials $\left(V_{3}^{h}\right)_{l \pm}$, which we identify with the effective hadronic potentials $V_{l \pm}^{h}$ for $\pi^{+} p$ scattering, we have new potentials $\left(V_{1}^{h}\right)_{l \pm}$ which are constructed in order to reproduce the phase shifts $\left(\delta_{1}^{h}\right)_{l \pm}$, using the same RSEs containing the masses $m_{p}$ and $\mu_{c}$. We therefore have $2 \times 2$ effective hadronic potential matrices $\mathbf{V}_{l \pm}^{h}$ which are isospin invariant:

$$
\mathbf{V}_{l \pm}^{h}=\mathbf{O}\left(\phi^{h}\right)\left(\begin{array}{cc}
\left(V_{1}^{h}\right)_{l \pm} & 0 \\
0 & \left(V_{3}^{h}\right)_{l \pm}
\end{array}\right) \mathbf{O}\left(\phi^{h}\right)^{t}
$$

The electromagnetic correction matrices are obtained by adding electromagnetic potential matrices $\mathbf{V}_{l \pm}^{e m}$ to the $\mathbf{V}_{l \pm}^{h}$ and then using these total potential matrices in coupled RSEs that model the physical situation. Full details will be given in Section 3. The other constraint that we impose on the effective hadronic potential matrices in Eq. (3) is that they be energy independent. This assumption was already made for the hadronic potentials used in the calculations for $\pi^{+} p$ elastic scattering described in Ref. [1]. Isospin invariance identifies these as the potentials $\left(V_{3}^{h}\right)_{l \pm}$ and we now require the new potentials $\left(V_{1}^{h}\right)_{l \pm}$ to be energy independent as well.

As we remarked in Ref.[1], the hadronic potential matrices in the absence of the electromagnetic interaction would be different from the effective matrices in its presence. Therefore isospin invariance for the $\mathbf{V}_{l \pm}^{h}$ (Eq.(3)) is a separate assumption. However, in the absence of any reliable model results for what 
happens when the electromagnetic interaction is switched on, in order to analyse the $\pi^{-} p$ scattering data we have no choice but to make this assumption and to test it by seeing if it is possible to obtain a statistically acceptable fit to the data. Implicit in this assumption is the requirement that the $T=3 / 2$ phase shifts for the $\pi^{-} p$ analysis be fixed at their values from the $\pi^{+} p$ analysis. To allow the possibility of different ' $T=3 / 2$ ' phase shifts here would already introduce the violation of isospin invariance, which is exactly what we wish to avoid if possible. A specific model for this violation would then be needed for a meaningful PSA to be possible, and a modified formalism would need to be used.

We tested the assumption of isospin invariance at the effective hadronic level and of energy independent effective hadronic potentials by first of all analysing only the $\pi^{-} p$ elastic scattering data, which are far more extensive than the charge exchange data. To anticipate results to be given later, a PSA based on these assumptions gives a fit to the present $\pi^{-} p$ elastic scattering data that is statistically poor but just acceptable. If the accumulation of data eventually results in a clearly unacceptable fit, for which there are systematic deviations of the data from the fitted values, this would already be evidence of 'dynamical' violation of isospin invariance (that is, beyond the effect of the electromagnetic interaction and the mass differences). The PSA and the calculation of the electromagnetic corrections would then need to be reconsidered. As things stand at present, the data on $\pi^{ \pm} p$ elastic scattering is consistent with the assumption of isospin invariance of the effective hadronic interaction and of energy independent hadronic potentials for calculating the electromagnetic corrections. This does not provide evidence for these assumptions; an investigation of the possibility of dynamical violation of isospin invariance requires the study of the data on $\pi^{-} p$ charge exchange scattering and on pionic hydrogen. Some positive evidence from the former is given in Refs. [4] and [5]. We will reconsider all of the evidence in a later paper concerned with a phase shift analysis of low energy $\pi^{ \pm} p$ scattering data and the comparison of the $s$-wave scattering lengths obtained from this analysis and from the position and width of the $1 s$ level of pionic hydrogen. This paper will complete our study of the pion-nucleon interaction at low energies.

The basic ideas sketched in this introduction will be fully developed in the rest of the paper. Section 2 will set out the formalism for the scattering amplitude matrices in the two-channel case, while Section 3 will give the method of calculating the electromagnetic correction matrices for the $s-, p_{1 / 2^{-}}$and $p_{3 / 2^{-}}$ waves. The numerical results for these corrections will be given in Section 4. 


\section{Scattering formalism}

We begin by writing the $2 \times 2$ matrices of no-flip and spin-flip scattering amplitudes for the $\left(\pi^{-} p, \pi^{0} n\right)$ system in the form

$$
\begin{gathered}
\mathbf{f}=\mathbf{f}^{e m}+\sum_{l=0}^{\infty}\left\{(l+1) \mathbf{e}_{l+} \mathbf{f}_{l+} \mathbf{e}_{l+}+l \mathbf{e}_{l-} \mathbf{f}_{l-} \mathbf{e}_{l-}\right\} P_{l}, \\
\mathbf{g}=\mathbf{g}^{e m}+i \sum_{l=1}^{\infty}\left(\mathbf{e}_{l+} \mathbf{f}_{l+} \mathbf{e}_{l+}-\mathbf{e}_{l-} \mathbf{f}_{l-} \mathbf{e}_{l-}\right) P_{l}^{1}
\end{gathered}
$$

where

$$
\begin{gathered}
\mathbf{e}_{l \pm}=\left(\begin{array}{cc}
\exp \left(i \Sigma_{l \pm}\right) & 0 \\
0 & 1
\end{array}\right) \\
\Sigma_{l \pm}=\left(\sigma_{l}-\sigma_{0}\right)+\sigma_{l}^{e x t}+\sigma_{l \pm}^{r e l}+\sigma_{l}^{v p} .
\end{gathered}
$$

The pieces of $\Sigma_{l \pm}$ are given by Eqs.(21-23) and (29) of Ref.[1], with a change of sign in each case. Eqs. $(4,5)$ are the obvious generalisations of Eqs. $(30,31)$ of Ref.[1]. The matrices $\mathbf{f}^{e m}, \mathbf{g}^{e m}$ are just

$$
\mathbf{f}^{e m}=\left(\begin{array}{cc}
f^{e m} & 0 \\
0 & 0
\end{array}\right), \mathbf{g}^{e m}=\left(\begin{array}{cc}
g^{e m} & 0 \\
0 & 0
\end{array}\right),
$$

where the electromagnetic amplitudes $f^{e m}, g^{e m}$ for $\pi^{-} p$ scattering have the same decomposition as for $\pi^{+} p$ scattering, namely

$$
\begin{gathered}
f^{e m}=f^{p c}+f_{1 \gamma E}^{e x t}+f_{1 \gamma E}^{r e l}+f^{v p}, \\
g^{e m}=g_{1 \gamma E}^{r e l} .
\end{gathered}
$$

The expressions for the components of $f^{e m}, g^{e m}$ are those given in Eqs.(7)-(9), (18) and (20) of Ref.[1], with $\alpha \rightarrow-\alpha, \eta \rightarrow-\eta$ in Eq.(18) and a change of sign in the other four amplitudes. The form factors are unchanged since $F_{\pi}$ is the same for $\pi^{+}$and $\pi^{-}$. 
The $2 \times 2$ matrices $\mathbf{f}_{l \pm}$ of partial wave amplitudes are written most conveniently in the form

$$
\begin{gathered}
\mathbf{f}_{l \pm}=\left(\begin{array}{cc}
q_{c}^{-1 / 2} & 0 \\
0 & q_{0}^{-1 / 2}
\end{array}\right) \mathbf{T}_{l \pm}^{n}\left(\begin{array}{cc}
q_{c}^{-1 / 2} & 0 \\
0 & q_{0}^{-1 / 2}
\end{array}\right) \\
\mathbf{T}_{l \pm}^{n}=\mathbf{t}_{l \pm}^{n}\left(\mathbf{1}_{2}-i \mathbf{t}_{l \pm}^{n}\right)^{-1}
\end{gathered}
$$

where $q_{c}$ is given by Eq.(10) of Ref.[1] and $q_{0}$ is the corresponding c.m. momentum for the $\pi^{0} n$ channel,

$$
q_{0}^{2}=\frac{\left[W^{2}-\left(m_{n}-\mu_{0}\right)^{2}\right]\left[W^{2}-\left(m_{n}+\mu_{0}\right)^{2}\right]}{4 W^{2}}
$$

$m_{n}$ and $\mu_{0}$ being the masses of the neutron and $\pi^{0}$ respectively. The nuclear matrices $\mathbf{t}_{l \pm}^{n}$ in Eq.(11) are those introduced in Section 1.

The expressions (10) and (11), with real symmetric matrices $\mathbf{t}_{l \pm}^{n}$, assume twochannel unitarity, that is the absence of any competing channels. This is not quite true, since the $\gamma n$ channel introduces inelastic corrections to the $\mathbf{T}_{l \pm}^{n}$ which need to be taken into account. In the energy range $T_{\pi} \leq 100 \mathrm{MeV}$ these corrections are almost insignificant and it is sufficient to use the results of Ref.[2], which are derived from known amplitudes for the reactions $\gamma n \rightarrow \pi^{-} p, \pi^{0} n$ using three-channel unitarity. It is unnecessary to introduce a complex hadronic potential matrix. The observables for $\pi^{-} p$ elastic and charge exchange scattering need to be calculated from the partial wave amplitudes $f_{c c}, f_{0 c}$ given by Eq.(10), with $T_{c c}^{n}, T_{0 c}^{n}$ replaced by $T_{c c}, T_{0 c}$, where

$$
T_{c c}=T_{c c}^{n}+\Delta T_{c c}^{\gamma n}, T_{0 c}=T_{0 c}^{n}+\Delta T_{0 c}^{\gamma n} .
$$

We have dropped the subscript $l \pm$ for convenience and have used the subscripts $c, 0$ to denote the channels $\pi^{-} p, \pi^{0} n$ respectively. The nuclear quantities $T_{c c}^{n}$, $T_{0 c}^{n}$ are calculated from Eq.(11) with the real symmetric matrix $\mathbf{t}^{n}$ given by Eq.(1). The corrections $\Delta T_{c c}^{\gamma n}, \Delta T_{0 c}^{\gamma n}$ (for the $s$ - and $p_{3 / 2}$-waves) are related to the quantities $\bar{\eta}_{1}, \bar{\eta}_{3}$ and $\eta_{13}$ in Table IV of Ref.[2] by the formulae

$$
\begin{aligned}
2 i \Delta T_{c c}^{\gamma n} & =-\frac{2}{3} \bar{\eta}_{1} \exp \left(2 i \delta_{1}^{h}\right)-\frac{1}{3} \bar{\eta}_{3} \exp \left(2 i \delta_{3}^{h}\right)-\frac{8}{9} \eta_{13} \exp \left\{i\left(\delta_{1}^{h}+\delta_{3}^{h}\right)\right\}, \quad(13) \\
2 i \Delta T_{0 c}^{\gamma n} & =\frac{\sqrt{2}}{3} \bar{\eta}_{1} \exp \left(2 i \delta_{1}^{h}\right)-\frac{\sqrt{2}}{3} \bar{\eta}_{3} \exp \left(2 i \delta_{3}^{h}\right)-\frac{2 \sqrt{2}}{9} \eta_{13} \exp \left\{i\left(\delta_{1}^{h}+\delta_{3}^{h}\right)\right\} .(14
\end{aligned}
$$

The decomposition of the matrices $\mathbf{t}_{l \pm}^{n}$ into a hadronic part $\mathbf{t}_{l \pm}^{h}$ and its electromagnetic correction $\mathbf{t}_{l \pm}^{e m}$ is given in Eq.(2). As we have discussed, the matrices 
$\mathbf{t}_{l \pm}^{h}$ refer to an effective hadronic situation which is isospin invariant and in which all the pions have the mass $\mu_{c}$ and the nucleons the mass $m_{p}$. The aim of our calculation is to obtain for the $s^{-}, p_{1 / 2^{-}}$and $p_{3 / 2^{-}}$waves the three independent elements of the symmetric correction matrix $\mathbf{t}_{l \pm}^{e m}$. However, the corrections in this form do not convey information in the way we would naturally like to have it. It is therefore customary to write the nuclear matrices in the form

$$
\mathbf{t}^{n}=\mathbf{O}(\phi)\left(\begin{array}{cc}
\tan \delta_{1}^{n} & 0 \\
0 & \tan \delta_{3}^{n}
\end{array}\right) \mathbf{O}(\phi)^{t}
$$

and to define three new corrections $C_{1}, C_{3}$ and $\Delta \phi$ by

$$
C_{1}=\delta_{1}^{n}-\delta_{1}^{h}, C_{3}=\delta_{3}^{n}-\delta_{3}^{h}, \Delta \phi=\phi-\phi^{h}
$$

Here $\phi$ is the mixing angle, which we choose to lie between 0 and $\pi / 2$. This convention then fixes the labelling of the eigenphases and ensures that $\tan \delta_{i}^{n}$ is close to $\tan \delta_{i}^{h}(i=1,3)$.

To proceed with the calculation of the corrections we need the potential matrices $\mathbf{V}_{l \pm}^{h}$ and $\mathbf{V}_{l \pm}^{e m}$ which appear in the coupled RSEs that lead to the nuclear matrices $\mathbf{t}_{l \pm}^{n}$. The effective hadronic potential matrices have the isospin invariant form of Eq.(3) and the potentials $V_{\alpha}^{h}(\alpha=1,3)$ are constructed so as to reproduce the hadronic phase shifts $\delta_{\alpha}^{h}$ via the RSEs

$$
\left(\frac{d^{2}}{d r^{2}}-\frac{l(l+1)}{r^{2}}+q_{c}^{2}-2 m_{c} f_{c} V_{\alpha}^{h}(r)\right) u_{\alpha}(r)=0
$$

The phase shifts are given by the asymptotic behaviour $\sin \left(q_{c} r-l \pi / 2+\delta_{\alpha}^{h}\right)$ of the regular wavefunctions. The quantities $q_{c}$ and $f_{c}$ are defined in Eq.(10) of Ref.[1] and $m_{c}$ is the reduced mass of the $\pi^{-} p$ system. The electromagnetic potential matrix $\mathbf{V}_{l \pm}^{e m}$ is

$$
\mathbf{V}_{l \pm}^{e m}=\left(\begin{array}{cc}
V_{l \pm}^{e m} & 0 \\
0 & 0
\end{array}\right)
$$

and $V_{l \pm}^{e m}$ has the decomposition given in Eq.(25) of Ref.[1]:

$$
V_{l \pm}^{e m}=V^{p c}+V^{e x t}+V_{l \pm}^{r e l}+V^{v p}
$$


The full potential matrix is

$$
\mathbf{V}_{l \pm}=\mathbf{V}_{l \pm}^{e m}+\mathbf{V}_{l \pm}^{h}
$$

The pieces of $V_{l \pm}^{e m}$ in Eq.(17), and therefore $V_{l \pm}^{e m}$ itself, have the opposite sign compared with the case of $\pi^{+} p$ scattering. (This is true for $V_{l \pm}^{r e l}$ since $\sigma_{l \pm}^{\text {rel }}$ is calculated only to order $\alpha$.) Full details of the parts of $V_{l \pm}^{e m}$ were given in Section 2 of Ref.[1].

\section{Evaluation of the corrections}

The partial wave RSEs for the two-channel case, which we use in order to model the physical situation, are given by the natural generalisation of Eq.(34) of Ref.[1]:

$$
\left\{\left(\frac{d^{2}}{d r^{2}}-\frac{l(l+1)}{r^{2}}\right) \mathbf{1}_{2}+\mathbf{Q}^{2}-2 \mathbf{m f V}_{l \pm}(r)\right\} \mathbf{u}_{l \pm}(r)=\mathbf{0}
$$

The full potential matrices $\mathbf{V}_{l \pm}$ have the form given in Eqs.(3,18-20). The matrices $\mathbf{Q}, \mathbf{m}$ and $\mathbf{f}$ are

$$
\mathbf{Q}=\left(\begin{array}{cc}
q_{c} & 0 \\
0 & q_{0}
\end{array}\right), \mathbf{m}=\left(\begin{array}{cc}
m_{c} & 0 \\
0 & m_{0}
\end{array}\right), \mathbf{f}=\left(\begin{array}{cc}
f_{c} & 0 \\
0 & f_{0}
\end{array}\right),
$$

where $q_{0}$ is defined in Eq.(11) and

$$
m_{0}=\frac{m_{n} \mu_{0}}{m_{n}+\mu_{0}}, f_{0}=\frac{W^{2}-m_{n}^{2}-\mu_{0}^{2}}{2 m_{0} W} .
$$

The only nonzero entry of the electromagnetic potential term $2 \mathbf{m f V}^{e m}$ is just $2 m_{c} f_{c} V^{e m}$, which is the same as for $\pi^{+} p$ but with the change of sign in $V^{e m}$. The particular form $2 \mathbf{m f V ^ { h }}$ of the hadronic potential term, with $\mathbf{V}^{h}$ a real symmetric energy independent matrix satisfying isospin invariance, is crucial for the calculation of the $\pi^{-} p$ electromagnetic corrections and we need to explain in detail why we chose it in this way. The issue is how to incorporate the physical mass differences into the two-channel problem. There is no guidance from the chain of reasoning that starts from the Bethe-Salpeter equation and proceeds via a three-dimensional reduction to integral equations for the partial wave amplitudes in momentum space, which contain a hadronic quasipotential. This method has been used for example in Ref.[6] to develop a dynamical 
model for low energy pion-nucleon scattering. Such a procedure ignores the mass differences and involves delicate issues like the choice of the reduction and the form factors at the vertices of the tree diagrams used. The partial wave quasipotentials, if converted to coordinate space, are nonlocal and energy dependent. Trying to incorporate the electromagnetic interaction and the mass differences into such a model in order to calculate the electromagnetic corrections would be impossible.

Since we are dealing with a spin 0 - spin $1 / 2$ system with the fermion much heavier than the boson, we are not far from the static limit and it is natural to look to the Klein-Gordon equation for guidance. For this equation with the potential $V^{p c}$ there is a simple transformation to an RSE with $\mu_{c}$ replaced by $\left(\mu_{c}^{2}+q_{c}^{2}\right)^{1 / 2}$, the static limit of $m_{c} f_{c}=\left(W^{2}-m_{p}^{2}-\mu_{c}^{2}\right) / 2 W$. The Appendix of Ref.[7] assumes that the hadronic potential enters the Klein-Gordon equation in the same way as $V^{p c}$, as the timelike component of a four-vector. It is then shown that the effective hadronic potential $V^{h}$ that appears in the resulting RSE must also be multiplied by $\left(\mu_{c}^{2}+q_{c}^{2}\right)^{1 / 2}$. However, it is also possible to introduce the hadronic potential as a scalar or as a combination of the two, so the assumption of Ref.[7], which is also made in the model of low energy pion-nucleon scattering of Ref.[8], requires further justification. Looking at $\pi^{+} p$ alone cannot decide anything, as we said in Ref.[1], but there is empirical evidence from the $\left(\pi^{-} p, \pi^{0} n\right)$ system for the choice made in Refs. [7,8].

At the hadronic level, with mass differences included, a study of the extrapolation of the invariant amplitudes to the Cheng-Dashen point favours this choice [9]; this provides some justification for the model used in Ref.[8]. As shown in Ref.[7], this implies that the hadronic potential term should have the form $2 \mathbf{m f V}^{h}$ given in Eq.(21). Writing this term in full is instructive; it is

$$
\left(\begin{array}{cc}
2 m_{c} f_{c}\left(\frac{2}{3} V_{1}^{h}+\frac{1}{3} V_{3}^{h}\right) & 2 m_{c} f_{c} \frac{\sqrt{2}}{3}\left(V_{3}^{h}-V_{1}^{h}\right) \\
2 m_{0} f_{0} \frac{\sqrt{2}}{3}\left(V_{3}^{h}-V_{1}^{h}\right) & 2 m_{0} f_{0}\left(\frac{1}{3} V_{1}^{h}+\frac{2}{3} V_{3}^{h}\right)
\end{array}\right) .
$$

It is then clear that introducing the energy dependent factors $f_{c}, f_{0}$ is not equivalent to having nonrelativistic reduced masses $m_{c}, m_{0}$ and energy dependent hadronic potentials for each isospin. The effect of the factors $f_{c}, f_{0}$ is large yet subtle; they change the way in which the violation of isospin invariance due to the mass differences is introduced. We studied the effect of choosing the hadronic potential term as $2 \mathbf{m} \tilde{\mathbf{V}}^{h}$, without the factor $\mathbf{f}$ and with $\tilde{\mathbf{V}}^{h}$ energy independent and isospin invariant. However, this resulted in large changes to some of the electromagnetic corrections. The most dramatic effect was on $C_{3}$ for the $p_{3 / 2}$-wave, which became much smaller; it changed sign near $70 \mathrm{MeV}$ and was $-0.4^{\circ}$ at $100 \mathrm{MeV}$, compared with $+0.5^{\circ}$ obtained with the choice $2 \mathbf{m f V}^{h}$ of the hadronic potential term in Eq.(21). The NORDITA value [2] at this energy is $+0.85^{\circ}$. The correction given by the potential model without 
the factor $\mathbf{f}$ is therefore completely at variance with that given by NORDITA. Their calculation did take account of the mass differences, though it is impossible to recover from their references exactly how this was done.

With the electromagnetic corrections calculated using the factor $\mathbf{f}$ in the hadronic potential term, the value of $\chi^{2}$ for the fit to 224 data points for $\pi^{-} p$ elastic scattering is 471.0 . When the factor $\mathbf{f}$ is absent, $\chi^{2}$ increases to 484.8 , so the fit becomes worse. Most of the increase comes from the data near $100 \mathrm{MeV}$, where the value of $C_{3}$ for the $p_{3 / 2}$-wave changes so much when the factor $\mathbf{f}$ is absent. By the usual statistical criteria, both fits are extremely poor, so once again decisive evidence is elusive. The large values of $\chi^{2}$ come from the data base itself. For the fit with the factor $\mathbf{f}$ included, there is no evidence of any systematic deviation, with either angle or energy, of the data points from the fitted curves. The deviations are erratic and it seems clear that in many cases the errors, particularly the systematic errors, have been underestimated. In the sense of giving a reasonable averaging over an internally inconsistent body of data, the fit with the factor $\mathbf{f}$ included is certainly better than that where this factor is absent. In summary, we have given three pieces of evidence that favour the inclusion of the specific energy dependence introduced by the factor $\mathbf{f}$ in the hadronic potential term: the extrapolation of the invariant amplitudes to the Cheng-Dashen point, the comparison with the results of NORDITA [2] (who also include the mass differences) and the better fit to the data (judged not only by the value of $\chi^{2}$ but also by the systematic deviation near $100 \mathrm{MeV}$ when $\mathbf{f}$ is not included).

We turn now to some calculational details. The RSEs (21) are integrated outwards from $r=0$ to obtain two linearly independent regular solution vectors. The integration proceeds to a distance $R$ (around $1000 \mathrm{fm}$ ) where the only part of the potential matrix that is not negligible is $V^{p c}$, which appears in $V_{c c}$. The components $V_{0 c}$ and $V_{00}$ of $\mathbf{V}$, which contain only linear combinations of the hadronic potential, become negligible beyond distances of a few fm, so the integration as far as $r=R$ is necessary only for the first component of $\mathbf{u}_{l \pm}$.

The matching at $r=R$, which leads to the matrix $\mathbf{t}_{l \pm}^{n}$, requires particular attention. In the $\pi^{-} p$ channel the matching is to a linear combination of the standard point charge Coulomb wavefunctions $F_{l}\left(-\eta f_{c} ; q_{c} r\right)$ and $G_{l}\left(-\eta f_{c} ; q_{c} r\right)$ and in the $\pi^{0} n$ channel it is to a linear combination of the free particle wavefunctions $q_{0} r j_{l}\left(q_{0} r\right)$ and $q_{0} r n_{l}\left(q_{0} r\right)$. To make the notation less complicated, we drop the subscripts $l \pm$ and $l$ for the moment and form the matrix $\mathbf{u}(r)$ :

$$
\mathbf{u}(r)=\left(\begin{array}{cc}
\left(\mathbf{u}^{(1)}(r)\right)_{c} & \left(\mathbf{u}^{(2)}(r)\right)_{c} \\
\left(\mathbf{u}^{(1)}(r)\right)_{0} & \left(\mathbf{u}^{(2)}(r)\right)_{0}
\end{array}\right)
$$


where $\mathbf{u}^{(i)}, i=1,2$, are two linearly independent solution vectors. For $r>R$, $\mathbf{u}(r)$ has the form

$$
\mathbf{u}(r)=\mathbf{m}^{1 / 2} \mathbf{f}^{1 / 2} \mathbf{Q}^{-1 / 2}(\hat{\mathbf{f}}(r) \mathbf{a}+\hat{\mathbf{g}}(r) \mathbf{b}),
$$

where

$$
\begin{gathered}
\hat{\mathbf{f}}(r)=\left(\begin{array}{cc}
\hat{f}_{c}(r) & 0 \\
0 & \hat{f}_{0}(r)
\end{array}\right), \hat{\mathbf{g}}(r)=\left(\begin{array}{cc}
\hat{g}_{c}(r) & 0 \\
0 & \hat{g}_{0}(r)
\end{array}\right), \\
\hat{f}_{c}(r)=\cos (\Delta \sigma) F\left(-\eta f_{c} ; q_{c} r\right)+\sin (\Delta \sigma) G\left(-\eta f_{c} ; q_{c} r\right), \\
\hat{g}_{c}(r)=\cos (\Delta \sigma) G\left(-\eta f_{c} ; q_{c} r\right)-\sin (\Delta \sigma) F\left(-\eta f_{c} ; q_{c} r\right), \\
\hat{f}_{0}(r)=r j\left(q_{0} r\right), \hat{g}_{0}(r)=r n\left(q_{0} r\right) .
\end{gathered}
$$

The particular forms of $\hat{f}_{c}(r), \hat{g}_{c}(r)$ in Eqs. $(27,28)$ arise from the choice of the additive electromagnetic amplitudes $(f, g)^{e m}$, which contain the parts $(f, g)^{e x t}$, $(f, g)^{r e l}$ and $(f, g)^{v p}$, and of the electromagnetic phase shifts $\Sigma_{l \pm}$, which contain $\Delta \sigma_{l \pm}=\sigma_{l}^{e x t}+\sigma_{l \pm}^{r e l}+\sigma_{l}^{v p}$. This means that, in order to obtain the correct matrices $\mathbf{t}_{l \pm}^{n}$ as defined in Eq.(10), it is necessary to match to the linear combinations of the point charge Coulomb wavefunctions given in Eqs. $(27,28)$, which correspond to the phase shift $\sigma_{l}+\Delta \sigma_{l \pm}$. The nuclear matrices $\mathbf{t}_{l \pm}^{n}$ are given by

$$
\mathbf{t}_{l \pm}^{n}=\mathbf{b}_{l \pm} \mathbf{a}_{l \pm}^{-1}
$$

The factor $\mathbf{m}^{1 / 2} \mathbf{f}^{1 / 2} \mathbf{Q}^{-1 / 2}$ in Eq.(25) appears naturally if one works with a symmetric matrix $\mathbf{t}^{n}$, as shown in Section 3 of Ref.[11]. Eq.(30) is the generalisation to two channels of the one-channel result given in Eq.(40) of Ref.[1].

The machinery for an iterative procedure exactly like that described in Ref.[1] for the $\pi^{+} p$ case has now been fully explained. The details of the PSA of the $\pi^{-} p$ elastic scattering data will be given in a separate paper. The hadronic phase shifts $\delta_{3}^{h}$ were fixed throughout the $\pi^{-} p$ PSA at the final values from the $\pi^{+} p$ PSA. The starting point for the $T=1 / 2$ hadronic phase shifts was the values from the analysis of Arndt et al. [12]. The parametric form of the $T=1 / 2$ hadronic potentials was taken to be the same as that used for the hadronic potentials in Ref.[1]. In Fig.1 we show these potentials for the final step of the iteration. At each step of the iteration the matrices $\mathbf{t}_{0+}^{e m}, \mathbf{t}_{1-}^{e m}$ and $\mathbf{t}_{1+}^{e m}$ were calculated using Eqs.(30),(1) and (2). The conversion to the corrections in the form $C_{1}, C_{3}$ and $\Delta \phi$ defined in Eq.(16) was done after the final values of these three matrices had been obtained. 


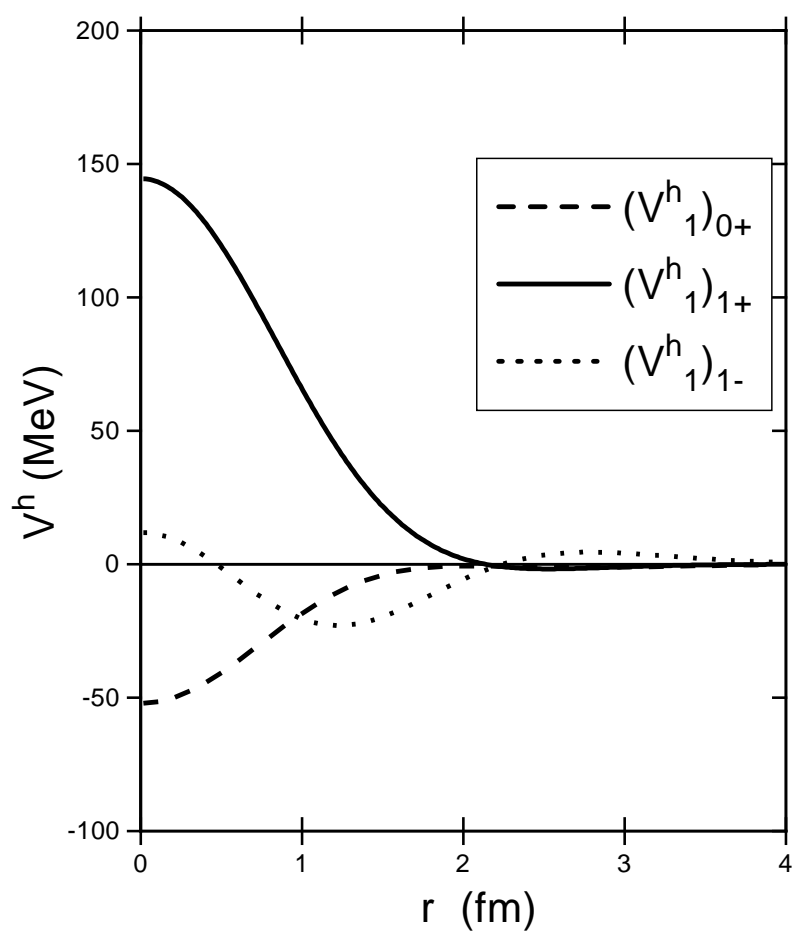

Fig. 1 . The $T=1 / 2$ hadronic potentials $\left(V_{1}^{h}\right)_{0+}$ and $\left(V_{1}^{h}\right)_{1 \pm}$.

\section{Results for the corrections}

In Tables 1, 2 and 3 we give the results for the electromagnetic corrections, in the case of the $s^{-}, p_{3 / 2^{-}}$and $p_{1 / 2^{-}}$waves respectively, in the form of the corrections $C_{1}$ and $C_{3}$ to the hadronic phase shifts and the correction $\Delta \phi$ to the isospin invariant mixing angle. They are given at $5 \mathrm{MeV}$ intervals from $T_{\pi}=10 \mathrm{MeV}$ to $T_{\pi}=100 \mathrm{MeV}$. The estimated uncertainties in the corrections are also given in the tables. They were obtained in the same way as the uncertainties in Table 1 of Ref. [1], by varying both the hadronic phase shifts used as input and the range parameter in the hadronic potentials. The only case for which the uncertainty in $C_{3}$ or $C_{1}$ is comparable with the error in the corresponding hadronic phase shift is $C_{3}$ for the $p_{3 / 2}$-wave around $85 \mathrm{MeV}$, where the uncertainty in $C_{3}$ is $0.028^{\circ}$ compared with an error in the phase shift of just over $0.03^{\circ}$.

Inspection of the RSEs in Eq.(21) shows that the electromagnetic corrections may be separated into two parts, one due to the inclusion of $\mathbf{V}^{e m}$ as an addition 
Table 1

Values in degrees of the $s$-wave electromagnetic corrections $C_{3}, C_{1}$ and $\Delta \phi$ as functions of the pion lab kinetic energy $T_{\pi}$ (in $\mathrm{MeV}$ ).

\begin{tabular}{|c|c|c|c|}
\hline$T_{\pi}$ & $C_{3}$ & $C_{1}$ & $\Delta \phi$ \\
\hline 10 & $-0.199 \pm 0.007$ & $0.208 \pm 0.002$ & $0.274 \pm 0.013$ \\
15 & $-0.175 \pm 0.008$ & $0.163 \pm 0.002$ & $0.116 \pm 0.011$ \\
20 & $-0.161 \pm 0.009$ & $0.133 \pm 0.001$ & $0.039 \pm 0.011$ \\
25 & $-0.151 \pm 0.010$ & $0.111 \pm 0.001$ & $-0.002 \pm 0.010$ \\
30 & $-0.145 \pm 0.010$ & $0.093 \pm 0.001$ & $-0.025 \pm 0.010$ \\
35 & $-0.140 \pm 0.011$ & $0.080 \pm 0.002$ & $-0.038 \pm 0.010$ \\
40 & $-0.136 \pm 0.011$ & $0.069 \pm 0.002$ & $-0.044 \pm 0.010$ \\
45 & $-0.134 \pm 0.011$ & $0.060 \pm 0.003$ & $-0.046 \pm 0.009$ \\
50 & $-0.132 \pm 0.012$ & $0.052 \pm 0.003$ & $-0.045 \pm 0.009$ \\
55 & $-0.130 \pm 0.012$ & $0.046 \pm 0.003$ & $-0.043 \pm 0.009$ \\
60 & $-0.128 \pm 0.013$ & $0.041 \pm 0.003$ & $-0.040 \pm 0.008$ \\
65 & $-0.127 \pm 0.014$ & $0.037 \pm 0.003$ & $-0.036 \pm 0.008$ \\
70 & $-0.126 \pm 0.015$ & $0.033 \pm 0.002$ & $-0.032 \pm 0.007$ \\
75 & $-0.124 \pm 0.015$ & $0.031 \pm 0.002$ & $-0.027 \pm 0.006$ \\
80 & $-0.123 \pm 0.016$ & $0.028 \pm 0.002$ & $-0.023 \pm 0.005$ \\
85 & $-0.122 \pm 0.018$ & $0.026 \pm 0.003$ & $-0.019 \pm 0.005$ \\
90 & $-0.120 \pm 0.019$ & $0.024 \pm 0.005$ & $-0.015 \pm 0.004$ \\
95 & $-0.118 \pm 0.020$ & $0.022 \pm 0.006$ & $-0.011 \pm 0.003$ \\
100 & $-0.117 \pm 0.021$ & $0.021 \pm 0.007$ & $-0.007 \pm 0.002$ \\
\hline
\end{tabular}

to $\mathbf{V}^{h}$ (Eq.(20)) and the other due to the difference between the masses of the particles in the two channels $\left(q_{c} \neq q_{0}, m_{c} \neq m_{0}, f_{c} \neq f_{0}\right)$. The marked increase in $\Delta \phi$ for small $T_{\pi}$ is due to the latter effect. In Ref.[1] we have also decomposed our results into the contributions coming from the separate pieces of $V^{e m}$ as given in Eq.(19). Giving this decomposition in the coupled channel case would involve us in complicated notation and would not convey any useful information: the relative importance of the single pieces varies with the partial wave, with the energy and with the particular correction $\left(C_{1}, C_{3}\right.$ or $\left.\Delta \phi\right)$.

Comparison with the results of the NORDITA group [2] for the $s$ - and $p_{3 / 2^{-}}$ waves is complicated by the different quantities $\left(\Delta_{1}, \Delta_{3}, \Delta_{13}\right)$ that they use 


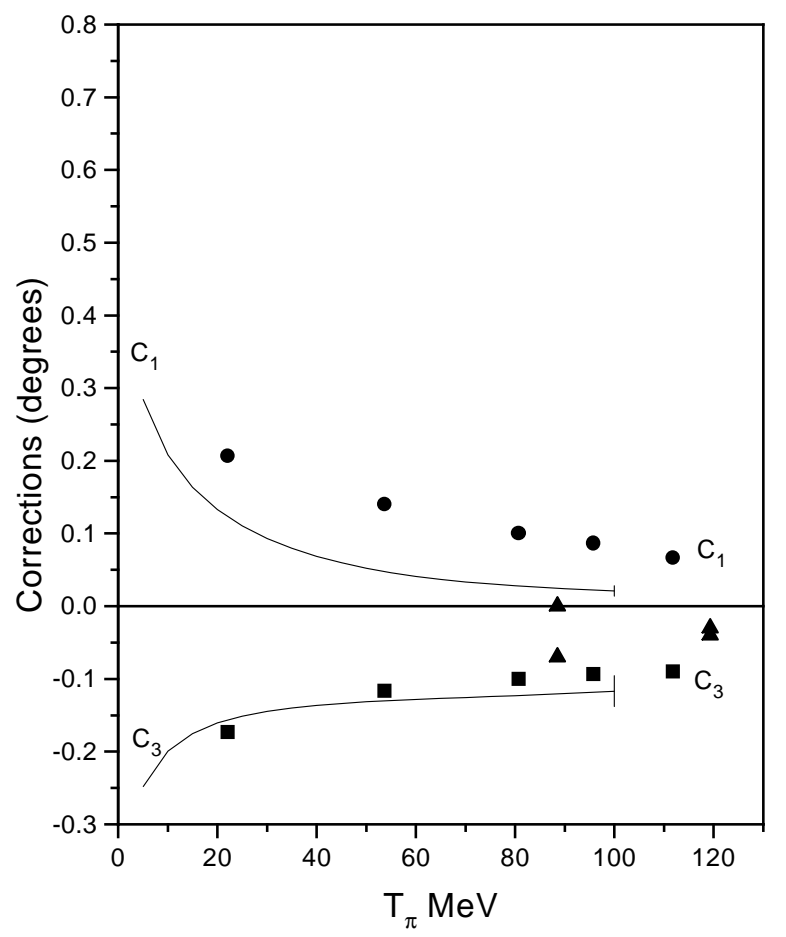

Fig. 2. Values in degrees of the electromagnetic corrections $C_{1}$ and $C_{3}$ for the $s$-wave from our present calculation (solid curves), from NORDITA [2] (circles) and from Zimmermann [10] (triangles).

for the corrections. The relation with our corrections is

$$
\Delta_{1}=-\frac{3}{2} C_{1}, \Delta_{3}=-3 C_{3}, \Delta_{13}=\frac{3}{\sqrt{2}} \Delta \phi \sin \left(\delta_{1}^{n}-\delta_{3}^{n}\right),
$$

the last relation being valid for $\Delta \phi$ very small. Our results are compared with those of NORDITA [2] and Zimmermann [10] in Figs. 2 and 3 for the s-wave and Figs. 4 and 5 for the $p_{3 / 2}$-wave. We have indicated in Figs. $2-5$ the uncertainties in our corrections at $100 \mathrm{MeV}$, as given in Tables 1 and 2. No errors are given in Refs. [2] and [10] for the corrections presented there. The most important differences are in $\Delta \phi$ for the s-wave and in $C_{3}$ for the $p_{3 / 2^{-}}$ wave. The former is probably due to differences in the treatment of the mass differences, the latter to the neglect in the NORDITA calculations of medium and short range effects due to $t$ - and $u$-channel exchanges. The calculation of the $\pi^{-} p$ corrections is not on as firm ground as the calculation of those for $\pi^{+} p$. It is difficult to judge the treatment of the mass differences in the NORDITA 


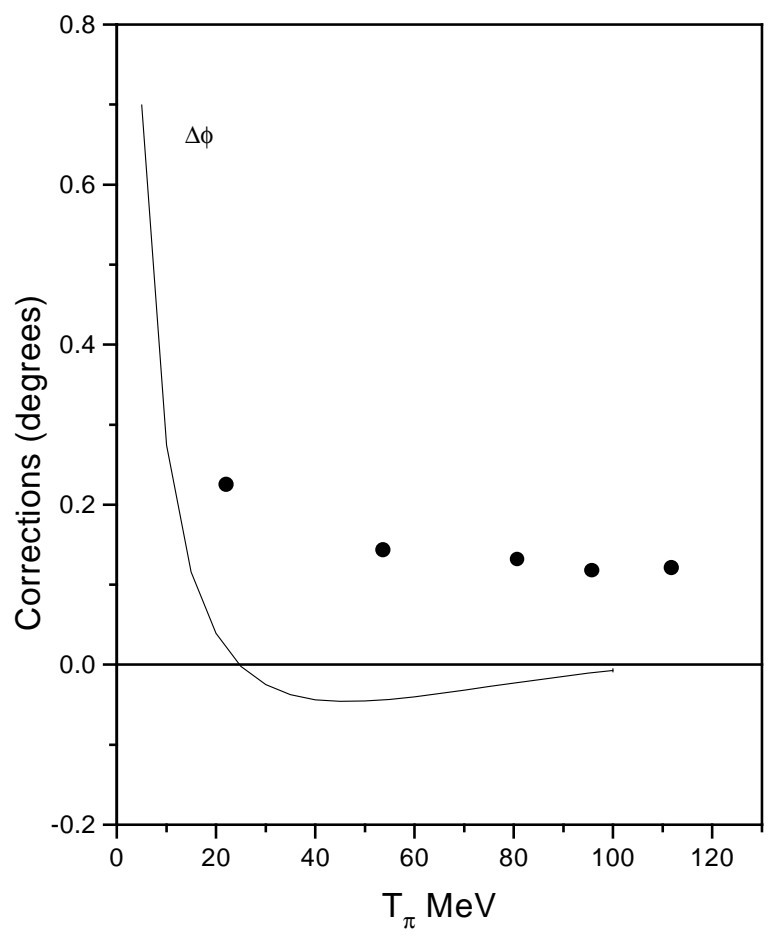

Fig. 3. Values in degrees of the electromagnetic correction $\Delta \phi$ for the $s$-wave from our present calculation (solid curve), from NORDITA [2] (circles) and from Zimmermann [10] (triangles).

calculations because so little detail is given in Ref. [3], while Ref. [10] has a double energy dependence of the hadronic potential term (a factor which is almost the same as our $\mathbf{f}$ at low energies plus energy dependent potentials). We have remedied the obvious deficiencies in these calculations and claim that as a result the values of the corrections given in Tables 1 to 3 are more reliable.

The corrections for the $p_{1 / 2}$-wave are given here for the first time. It turns out that for the analysis of the data they play a negligible role, due to the smallness of the hadronic phases in that partial wave.

The corrections in Tables 1-3 are intended for use in future PSAs of $\pi^{-} p$ scattering experiments, provided that these PSAs use Eqs.(4), (5), (15) and (16) and the inelasticity corrections due to the $\gamma n$ channel given in Eqs.(13) and (14). For very small angles or energies, corrections to the expressions we have given for $f^{v p}$ and $\sigma_{l}^{v p}$ may also be needed, as discussed in Section 2 of Ref. [1]. 
Table 2

Values in degrees of the $p_{3 / 2}$-wave electromagnetic corrections $C_{3}, C_{1}$ and $\Delta \phi$ as functions of the pion lab kinetic energy $T_{\pi}$ (in $\mathrm{MeV}$ ).

\begin{tabular}{|c|c|c|c|}
\hline$T_{\pi}$ & $C_{3}$ & $C_{1}$ & $\Delta \phi$ \\
\hline 10 & $0.159 \pm 0.001$ & $-0.002 \pm 0.000$ & $-4.715 \pm 0.049$ \\
15 & $0.187 \pm 0.001$ & $-0.003 \pm 0.000$ & $-3.044 \pm 0.038$ \\
20 & $0.209 \pm 0.002$ & $-0.005 \pm 0.000$ & $-2.146 \pm 0.030$ \\
25 & $0.229 \pm 0.003$ & $-0.007 \pm 0.000$ & $-1.592 \pm 0.025$ \\
30 & $0.246 \pm 0.003$ & $-0.009 \pm 0.000$ & $-1.222 \pm 0.020$ \\
35 & $0.261 \pm 0.003$ & $-0.011 \pm 0.000$ & $-0.961 \pm 0.015$ \\
40 & $0.276 \pm 0.002$ & $-0.013 \pm 0.000$ & $-0.769 \pm 0.009$ \\
45 & $0.290 \pm 0.002$ & $-0.016 \pm 0.000$ & $-0.623 \pm 0.010$ \\
50 & $0.305 \pm 0.005$ & $-0.018 \pm 0.001$ & $-0.511 \pm 0.014$ \\
55 & $0.320 \pm 0.009$ & $-0.021 \pm 0.001$ & $-0.422 \pm 0.018$ \\
60 & $0.336 \pm 0.012$ & $-0.023 \pm 0.001$ & $-0.351 \pm 0.021$ \\
65 & $0.352 \pm 0.015$ & $-0.026 \pm 0.001$ & $-0.293 \pm 0.023$ \\
70 & $0.370 \pm 0.019$ & $-0.029 \pm 0.001$ & $-0.246 \pm 0.024$ \\
75 & $0.390 \pm 0.023$ & $-0.031 \pm 0.001$ & $-0.207 \pm 0.024$ \\
80 & $0.410 \pm 0.026$ & $-0.034 \pm 0.001$ & $-0.174 \pm 0.024$ \\
85 & $0.433 \pm 0.028$ & $-0.037 \pm 0.002$ & $-0.145 \pm 0.024$ \\
90 & $0.456 \pm 0.028$ & $-0.039 \pm 0.002$ & $-0.121 \pm 0.023$ \\
95 & $0.481 \pm 0.025$ & $-0.042 \pm 0.002$ & $-0.100 \pm 0.024$ \\
100 & $0.506 \pm 0.017$ & $-0.045 \pm 0.003$ & $-0.082 \pm 0.025$ \\
\hline
\end{tabular}

\section{Acknowledgements}

We thank the Swiss National Foundation and PSI ('Paul Scherrer Institut') for financial support. We acknowledge very interesting discussions with W. R. Gibbs and we are indebted to two referees for several very helpful comments and suggestions.

\section{References}

[1] A. Gashi, E. Matsinos, G.C. Oades, G. Rasche and W.S. Woolcock, preceding paper. 


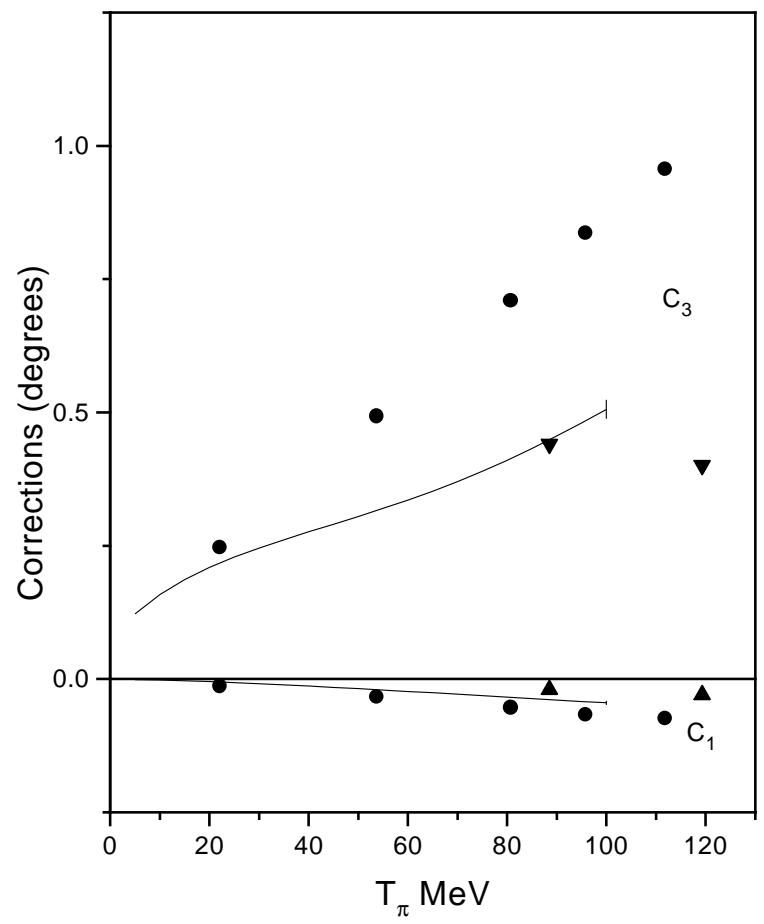

Fig. 4. Values in degrees of the electromagnetic corrections $C_{1}$ and $C_{3}$ for the $p_{3 / 2}$-wave from our present calculation (solid curves), from NORDITA [2] (circles) and from Zimmermann [10] (triangles).

[2] B. Tromborg, S. Waldenstrøm and I. Øverbø, Phys. Rev. D 15 (1977) 725.

[3] B. Tromborg, S. Waldenstrøm and I. Øverbø, Helv. Phys. Acta 51 (1978) 584.

[4] W.R. Gibbs, Li Ai and W.B. Kaufmann, Phys. Rev. Lett. 74 (1995) 3740.

[5] E. Matsinos, Phys. Rev. C 56 (1997) 3014.

[6] B.C. Pearce and B.K. Jennings, Nucl. Phys. A 528 (1991) 655.

[7] P.R. Auvil, Phys. Rev. D 4 (1971) 240.

[8] W.R. Gibbs, Li Ai and W.B. Kaufmann, Phys. Rev. C 57 (1998) 784.

[9] W.R. Gibbs, private communication.

[10] H. Zimmermann, Helv. Phys. Acta 48 (1975) 191.

[11] G. Rasche and W.S. Woolcock, Helv. Phys. Acta 45 (1976) 495. 


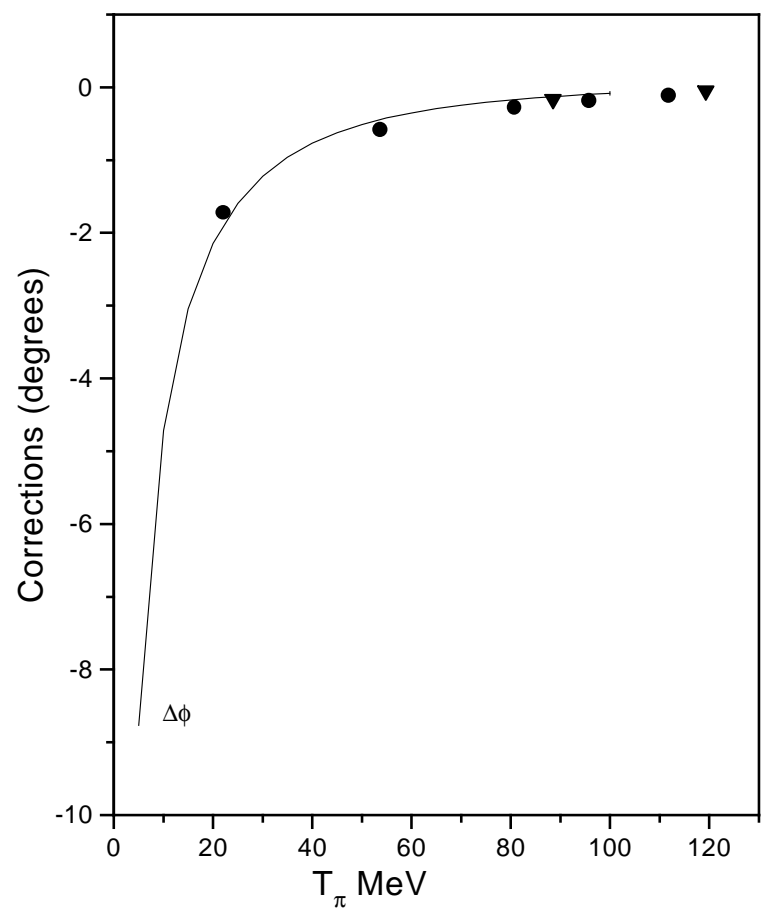

Fig. 5. Values in degrees of the electromagnetic correction $\Delta \phi$ for the $p_{3 / 2}$-wave from our present calculation (solid curve), from NORDITA [2] (circles) and from Zimmermann [10] (triangles).

[12] R.A. Arndt, R.L. Workman, I.I. Strakovsky and M.M. Pavan, " $\pi N$ Elastic Scattering Analyses and Dispersion Relation Constraints", nucl-th/9807087; R.A. Arndt and L.D. Roper, SAID on-line program. 
Table 3

Values in degrees of the $p_{1 / 2}$-wave electromagnetic corrections $C_{3}, C_{1}$ and $\Delta \phi$ as functions of the pion lab kinetic energy $T_{\pi}$ (in $\mathrm{MeV}$ ).

\begin{tabular}{|c|c|c|c|}
\hline$T_{\pi}$ & $C_{3}$ & $C_{1}$ & $\Delta \phi$ \\
\hline 10 & $-0.025 \pm 0.000$ & $-0.042 \pm 0.001$ & $15.911 \pm 0.052$ \\
15 & $-0.031 \pm 0.001$ & $-0.043 \pm 0.002$ & $10.308 \pm 0.053$ \\
20 & $-0.035 \pm 0.001$ & $-0.042 \pm 0.003$ & $7.288 \pm 0.052$ \\
25 & $-0.037 \pm 0.001$ & $-0.040 \pm 0.003$ & $5.448 \pm 0.050$ \\
30 & $-0.038 \pm 0.002$ & $-0.037 \pm 0.003$ & $4.225 \pm 0.048$ \\
35 & $-0.039 \pm 0.002$ & $-0.033 \pm 0.004$ & $3.360 \pm 0.048$ \\
40 & $-0.039 \pm 0.003$ & $-0.028 \pm 0.004$ & $2.720 \pm 0.050$ \\
45 & $-0.038 \pm 0.003$ & $-0.023 \pm 0.005$ & $2.230 \pm 0.054$ \\
50 & $-0.037 \pm 0.003$ & $-0.017 \pm 0.005$ & $1.844 \pm 0.063$ \\
55 & $-0.036 \pm 0.004$ & $-0.011 \pm 0.005$ & $1.530 \pm 0.072$ \\
60 & $-0.035 \pm 0.004$ & $-0.005 \pm 0.005$ & $1.268 \pm 0.083$ \\
65 & $-0.034 \pm 0.004$ & $0.002 \pm 0.005$ & $1.032 \pm 0.102$ \\
70 & $-0.032 \pm 0.004$ & $0.009 \pm 0.006$ & $0.910 \pm 0.105$ \\
75 & $-0.031 \pm 0.005$ & $0.015 \pm 0.005$ & $0.851 \pm 0.108$ \\
80 & $-0.030 \pm 0.007$ & $0.023 \pm 0.004$ & $0.784 \pm 0.110$ \\
85 & $-0.029 \pm 0.007$ & $0.030 \pm 0.004$ & $0.689 \pm 0.107$ \\
90 & $-0.027 \pm 0.007$ & $0.037 \pm 0.004$ & $0.572 \pm 0.108$ \\
95 & $-0.026 \pm 0.008$ & $0.043 \pm 0.005$ & $0.483 \pm 0.109$ \\
100 & $-0.025 \pm 0.008$ & $0.049 \pm 0.005$ & $0.411 \pm 0.108$ \\
\hline
\end{tabular}

